\title{
Fiber Bragg Grating Sensors and Sensor Arrays
}

\author{
Hartmut Bartelt \\ Institute of Photonic Technology, Albert-Einstein-Str. 9, 07745 Jena, Germany \\ hartmut.bartelt@ipht-jena.de
}

Keywords: fiber Bragg grating, grating sensors, grating sensor arrays

\begin{abstract}
Fiber Bragg gratings have found widespread application in sensor systems, e. g. for temperature, strain or refractive index measurements. The concept of fiber Bragg gratings allows also in a simple way the realisation of arrays of such sensors. The development of such optical fiber sensor systems often requires special fibers and grating structures which may go beyond more conventional Bragg grating structures in typical communication fibers. Concerning fibers there is, for example., a need of achieving fiber gratings in small diameter fibers and fiber tapers as well as in microstructured fibers. Special fiber grating structures are of interest e.g. in the visible wavelength range, which requires smaller spatial structures compared to more conventional gratings in the near infrared wavelength region. Examples for such modern developments in fiber Bragg grating technology for sensor applications will be presented and discussed.
\end{abstract}

\section{Introduction}

Fiber Bragg gratings (FBGs) have become standard elements in the development of new types of fiber optical sensors and sensor systems [1-2]. Standard techniques for making such fiber Bragg gratings are phase mask recording technique and the interferometric recording technique. Such gratings have been developed also for use in harsh environments like high temperatures [3]. Multiple gratings are of specific interest in sensor systems in order to allow quasi distributed sensing and for achieving sensor multiplexing. So called draw tower gratings, which are recorded by single pulses during the fiber drawing process, have been developed especially for this purpose [4-7]. These examples show the need for specific developments adapted to the needs of fiber sensor applications. Further special developments should therefore extend our capabilities to apply fiber Bragg gratings for additional sensing applications. We will discuss in the following such developments for improved and extended sensor applications such as:

- FBGs in small diameter fibers

- $\quad$ FBGs in fiber tapers

- $\quad$ FBGs for short wavelengths

- FBGs in microstructured fibers

\section{Fiber Bragg gratings in small diameter fibers}

Due to their small diameter, optical fibers are attractive sensor elements for embedding in structural materials. Examples are fiber reinforced thermoplastic composites. By embedding sensors, such as fiber Bragg gratings, into the material it becomes possible to monitor the structural behaviour during production and/or operation. For such applications it should be avoided that the composite structure is disturbed by the sensor element. Therefore smaller diameters of optical fibers than typically used in communication application are desirable. Special fibers for this purpose have been fabricated with fiber diameters of $80 \mathrm{~nm}$ for grating inscription during the fiber drawing process. It has been shown that also for such small diameter fibers efficient gratings can be achieved, which 
show similar properties to gratings in conventional sized fibers with a diameter of $125 \mu \mathrm{m}$ (fig. 1) (see also paper C-4: L16 [8]).
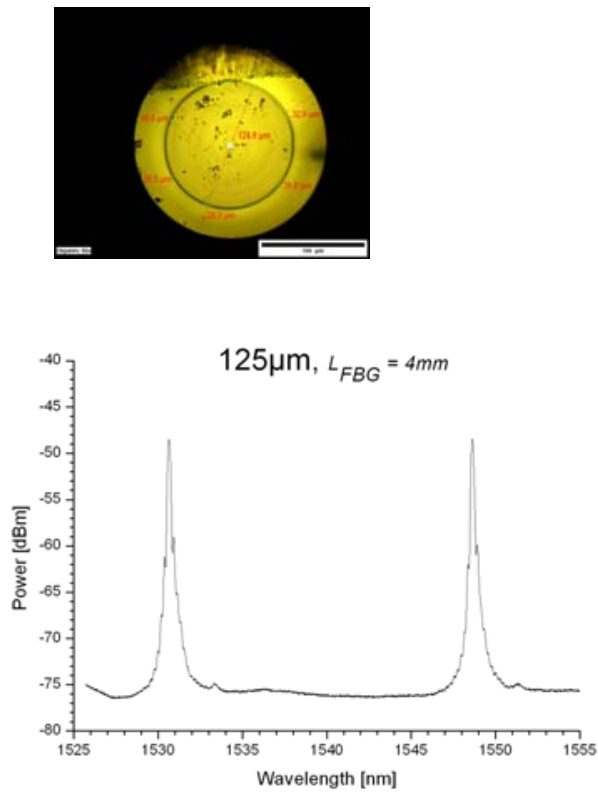
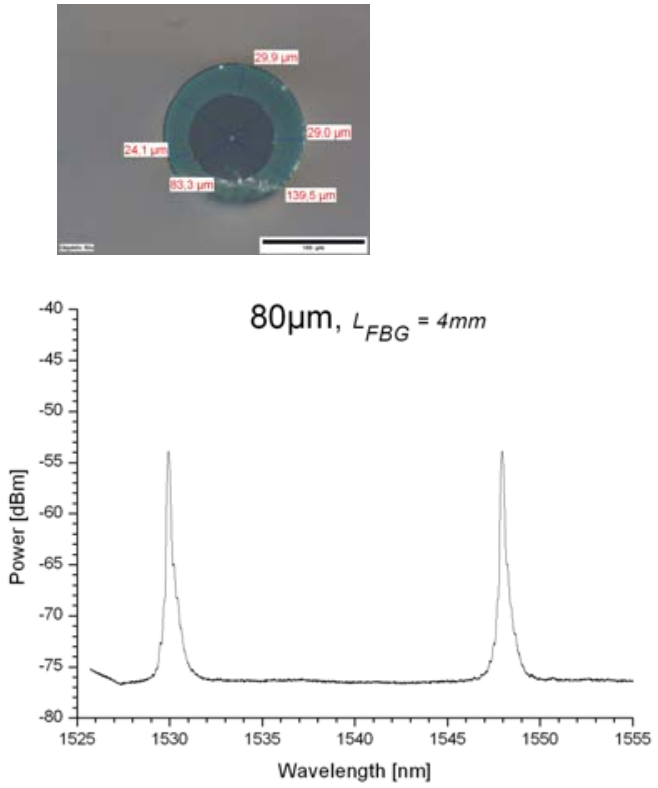

Fig. 1 Comparison of experimentally achieved fiber Bragg gratings in a conventional fiber with diameter of $125 \mu \mathrm{m}$ (left) and a fiber with diameter of $80 \mu \mathrm{m}$ (right)

\section{Fiber Bragg gratings in tapers}

The interaction of the guided light in a fiber with a FBG with the surrounding material can be used for a refractive sensor. In a more complex setup with metallized fibers also the effect of generating plasmonic waves can modify in a sensitive way the reflection properties for FBGs. Such effects require, however, an efficient overlapping of the guided light. For this purpose fibers with a small core diameter are needed with a reasonable extension of the evanescent wave in the medium to be measured. One possible approach are side polished fibers. In this case only in one orientation good overlapping of the guided light is obtained, which is a limiting factor for the achievable sensitivity. Optimized sensitivity can be therefore expected for tapered fiber structures with extremely small fiber cores. Typical techniques for making fiber tapers are drawing or etching technologies.

The concept for such a tapered sensor probe is shown in fig. 2. In the short tapered fiber end a fiber Bragg grating structure is inscribed. Additional layers of metal and high refractive index tantalpentoxide are applied to achieve increased field amplitudes at the core boundary and to shift the surface plasmon resonance to smaller refractive index values of the surrounding medium (adapted to water refractive index). This FBG is combined with a second FBG in the conventional part of the fiber for temperature measurements and temperature compensation. This FBG sensor element can now be used as a refractive sensor, where the refractive index value can be modified by specific biological binding reactions. Specific senor elements were made by an etching process achieving sensor tip diameters of a few micrometers and down to submicrometer dimensions. A result from a sensor probe with a diameter of $3 \mu \mathrm{m}$ is shown in figs. 3 and 4 .

Besides the good field overlapping such FBGs in tapered fibers also give the opportunity of small scale sensor nano probes. In a similar way it is possible to use tapers made by a taper drawing process. 


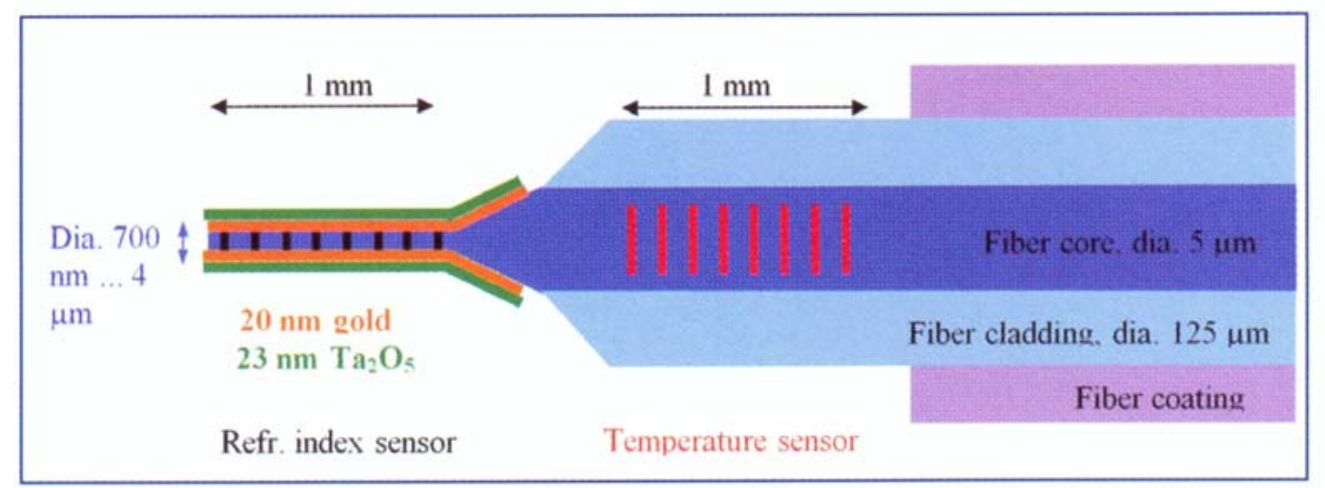

Fig. 2 Principle of the fiber grating probe for refractive sensing

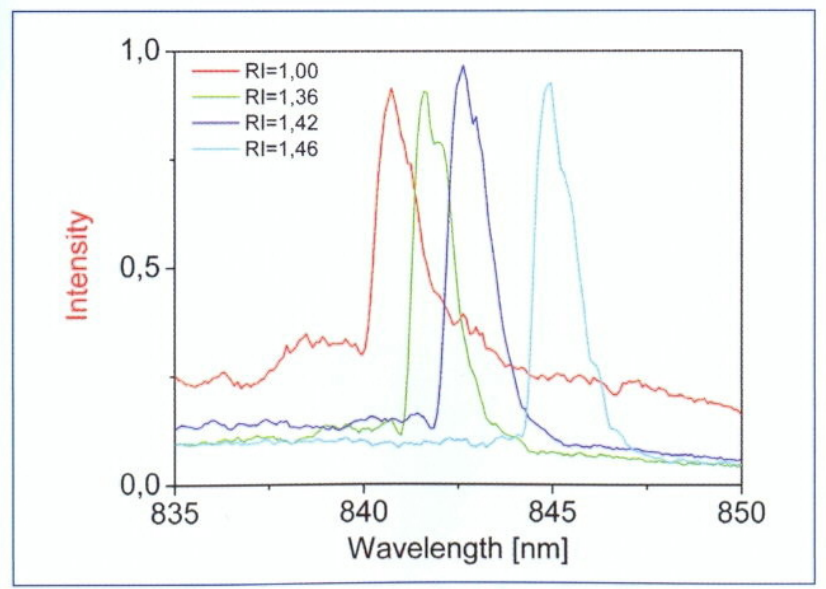

Fig. 3 Spectra of the FBG sensor probe with surrounding analytes of different refractive index

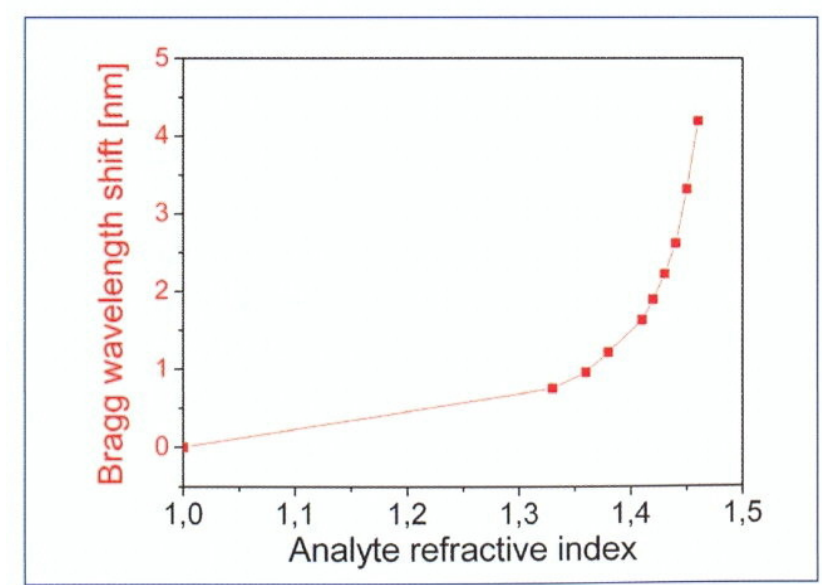

Fig. 4 Bragg wavelength shift depending on refractive index for a tapered fiber probe

\section{Fiber Bragg gratings for short wavelengths}

The typical wavelengths for fiber grating applications lie in the near infrared region corresponding to the mostly used wavelengths in waveguide optics. In order to cover new application fields, there is a growing interest to have available fiber Bragg gratings also for shorter wavelengths than the communication window wavelengths, especially in the visible wavelength range. However, the fabrication of such gratings presents special challenges due to the requirement of very small grating periods in the range of a few $100 \mathrm{~nm}$.

We use a Talbot interferometer for inscribing Bragg gratings, where we can change the spatial frequency of the Bragg gratings by a variation of the angle $\delta_{\mathrm{FBG}}$ of the interferometer mirrors. The Bragg wavelength $\lambda_{\mathrm{B}}$ is then a function of the Bragg period $\Lambda_{\mathrm{FBG}}$, the effective refractive index $\mathrm{n}_{\mathrm{eff}}$ in the fiber and the UV laser source wavelength $\lambda_{\mathrm{UV}}$ according to:

$$
\lambda_{B}=2 \cdot n_{\text {eff }} \cdot \Lambda_{F B G}=\frac{n_{\text {eff }} \cdot \lambda_{U V}}{\sin \vartheta_{F B G}} .
$$

By increasing the angle we have achieved to make FBGs down to a reflection wavelength of about $475 \mathrm{~nm}$ (fig. 5) in a non-polarization maintaining single mode fiber. A reflectivity of about 80\% with a spectral width smaller than $0.3 \mathrm{~nm}$ at an exposure time of $10 \mathrm{~min}$ was obtained. This wavelength value still does not represent the experimental limit of the setup, but was given by the 
available light source for observation (Xenon light source with a band edge at 460nm). A theoretical limit considering the used recording wavelength, the experimental setup and the increasing Fresnel reflections has been derived for a wavelength of about 360nm.

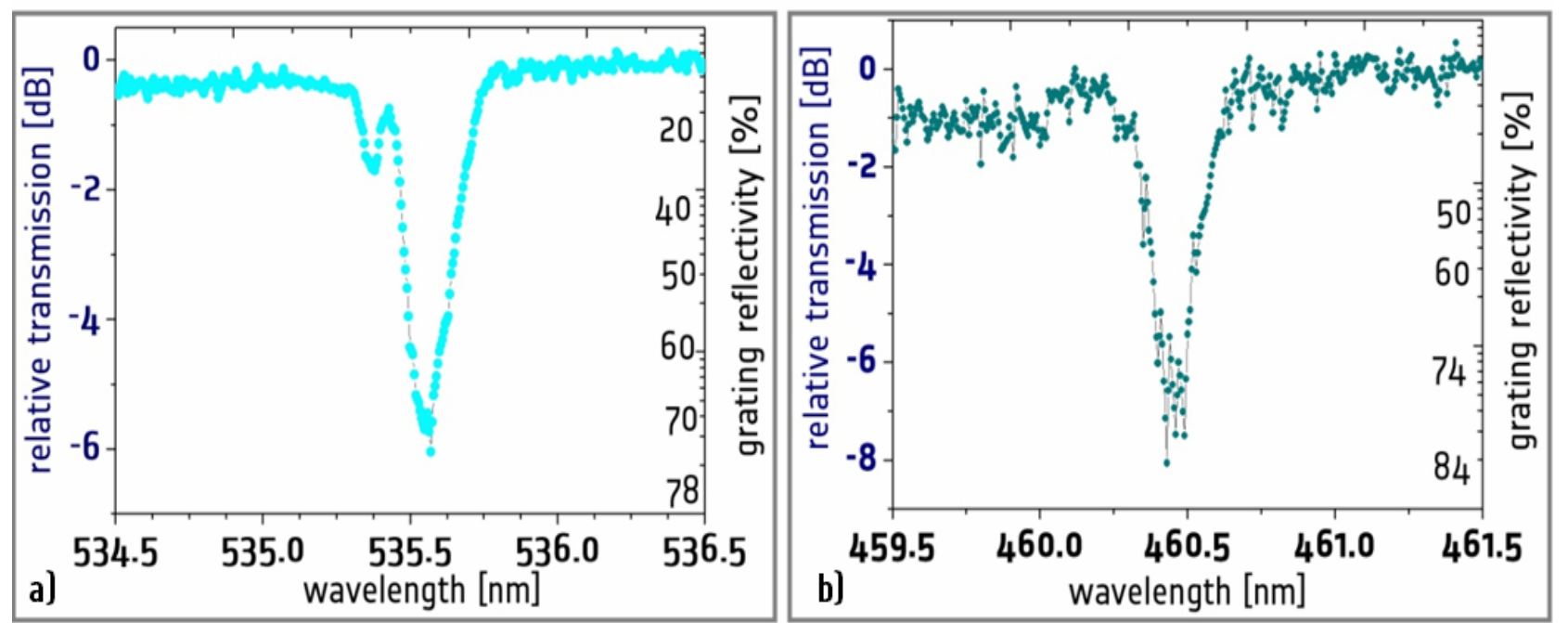

Fig. 5 (a) Short FBG with a length of less than $0.75 \mathrm{~mm}$; b) FBG with shortest measurable Bragg wavelength

The impressing high reflectivities, even for small fiber grating structures of about $200 \mathrm{~nm}$, can be explained from coupled wave theory. The required refractive index modulation of a grating is given by $[10,11]$ :

$$
\Delta n_{A C}=\frac{\operatorname{arctanh}(\sqrt{R}) \cdot \lambda_{B} \cdot\left(\lambda_{B}^{2}+\pi^{2} d^{2} N A^{2}\right)}{\pi^{3} d^{2} l_{F B G} N A^{2}} .
$$

For a constant grating length $\mathrm{l}_{\mathrm{FBG}}$ and a constant fiber diameter $\mathrm{d}$, a reduced index modulation is still sufficient for good reflectivity. The mode overlap factor increases for shorter wavelengths. At the same time, also the number of grating periods increases within the constant grating length. Both factors are helpful to achieve good reflectivity values.

\section{Fiber Bragg gratings in microstructured fibers (Photonic Crystal Fibers)}

Microstructured fibers with holey parts in the core and cladding region offer new possibilities for modifying the transmission properties of optical fibers. Usually, such fibers do not require doping of the fiber core for achieving guiding properties. Therefore the question arises how to make FBGs in such fibers $[9,10]$.

As an obvious way, it is possible to make such microstructured fibers with a specifically Ge-doped fiber core. In this case the doping would be not used for achieving guiding properties, but for obtaining a UV-sensitivity. The holey structure may eventually cause reflections and scattering effects. Such effects could make the inscription of efficient gratings difficult. But experimental results show, that indeed high reflectivivties can be achieved with conventional interferometric recording techniques. As an example, in fig. 6 FBG properties are shown for different gratings, which were produced in a microstructured fiber as shown in fig. 7 (air hole diameter and the pitch of the PCF are 3.5 and $4.2 \mu \mathrm{m}$, respectively). The gratings were written in the PCF by use of a 
248nm UV laser and a Talbot Interferometer. Two FBGs, $\mathrm{FBG}_{1}$ and $\mathrm{FBG}_{2}$, with a reflectivity of $35 \%$ and $33 \%$ are shown at the wavelength of $830.050 \mathrm{~nm}$ and $1080.063 \mathrm{~nm}$, respectively, before hydrogen loading with a $3 \mathrm{~dB}$ bandwidth of $0.148 \mathrm{~nm}$ and $0.165 \mathrm{~nm}$. A FBG with almost $100 \%$ reflectivity was achieved after the PCF was hydrogen-loaded for one week with a pressure of 200bar and at a temperature of $90^{\circ} \mathrm{C}$. For such highly photosensitive fibers several reflection peaks were observed near 830nm, resulting from the reflected higher-order modes. The gratings show similar sensitivities to temperature and strain as FBG in conventional solid fibers.

Figure 8 illustrates the temperature characteristics for the reflected wavelength within the temperature range from $7^{\circ} \mathrm{C}$ to $50^{\circ} \mathrm{C}$. As can be seen, the reflected wavelength was linearly shifted toward the longer wavelength with a sensitivity of $5.6 \mathrm{pm} /{ }^{\circ} \mathrm{C}$. The temperature dependency is well applicable as a temperature sensor.

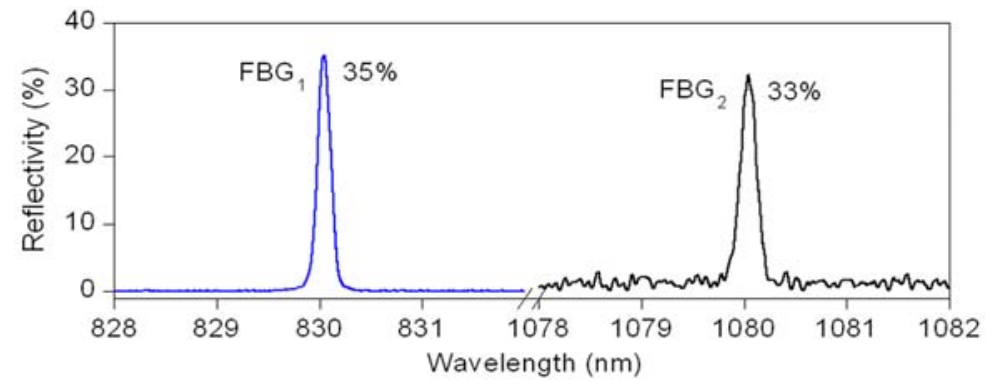

Fig. 6 Reflectivity of $\mathrm{FBG}_{1}$ and $\mathrm{FBG}_{2}$ written in PCF before hydrogen loading

Fig. 7 Cross section image of PCF 252b5 with air hole diameter of $3.5 \mu \mathrm{m}$ and pitch of $4.2 \mu \mathrm{m}$

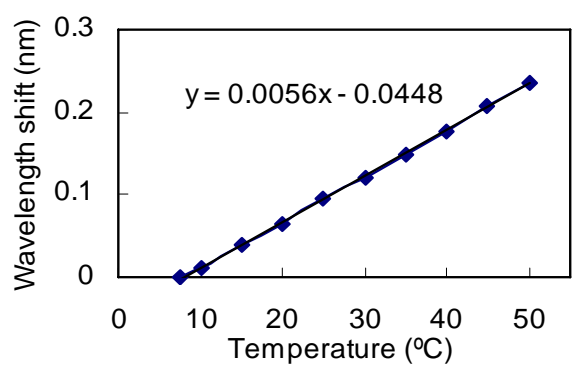

Fig. 8 Temperature characteristics of $\mathrm{FBG}_{1}$

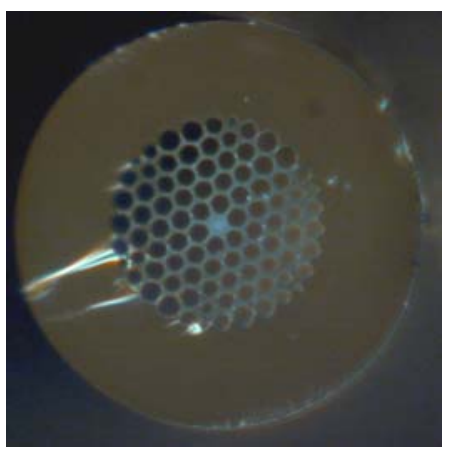

If no UV- sensitivity is provided in the fiber core, then other techniques have to be used for writing FBGs. The inscription with femtosecond laser pulses is such an option. We have therefore investigated the same microstructured fiber for FBG inscription with a femtosecond laser. Usually such lasers are used in the near infrared wavelength region. In our case we have used femtosecond laser pulses at a wavelength of 262nm in order to inscribe FBGs with high spatial resolution. At this wavelength also effects of the UV sensitivity can be expected. As shown in fig. 9 it is possible to achieve high reflectivities of about $90 \%$ (without hydrogen loading) in such fibers. The 3dBbandwidth was larger $(0.51 \mathrm{~nm})$ than for gratings recorded on the basis of the UV-sensitivity. 


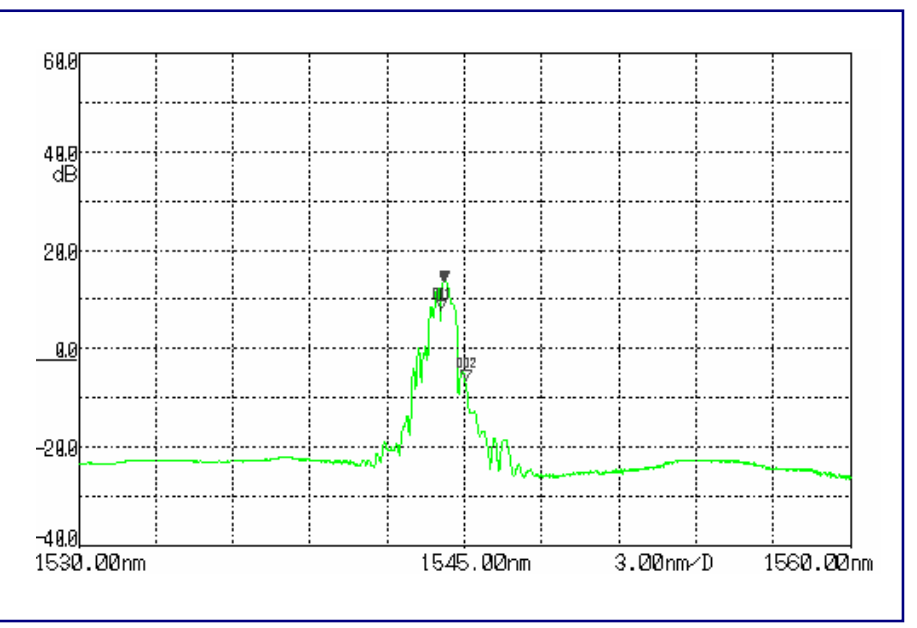

Fig.9 Wavelength dependent reflectivity of a FBG in a microstructured fiber according to fig.7 with femtosecond laser pulse inscription

\section{Conclusions}

Several techniques and concepts have been discussed which will allow to extend further the field of applications of FBGs. The great flexibility of such fiber integrated sensor probes concerning fiber types and dimensions underlines their growing potential in sensing applications. Additionally such flexibility will be of interest for further applications in information technology or for fiber laser resonators.

\section{Acknowledgements}

The contributions of Yinping Wang, Eric Lindner, Manfred Rothhardt, Michael Kautz and Eli Voet in the field of new types of fiber Bragg gratings are gratefully acknowledged. The presented work was funded in part by the Alexander von Humboldt Foundation and by the Thuringian Ministry TKM.

\section{References}

[1] Hill, O.H., Meltz, G., Fiber Bragg Grating Technology Fundamentals and Overview, Journal of Lightwave Technology 15(8), 1263-1276 (1997)

[2] Othonos, Andreas; Kalli, Kyriacos: Fiber Bragg Gratings: Fundamentals and Applications in Telecommunications and Sensing. Bd. 1. Artech House, 1999

[3] H. Bartelt, K. Schuster, S. Unger, C. Chojetzki, M. Rothhardt, I. Latka, Single-pulse fiber Bragg gratings and specific coatings for use at elevated temperatures, Appl. Optics 46 (17), 3417-3424 (2007)

[4] Askins, C.G., Tsai, T.-E., Williams, G.M., Putnam, M.A., Bashkansky, M. and Friebele, E.J., Fiber Bragg reflectors prepared by a single excimer pulse, Opt. Lett. 17(11), 833-835 (1992)

[5] Archambault, J.-L., Reekie, L., and Russell, P.St.J., 100\% reflectivity Bragg reflectors produced in optical fibres by single excimer laser pulses, Electron. Lett. 29(5), 453-455 (1993)

[6] Dong, L., J.L. Archambault, L. Reekie, P.St.J. Russell, and D.N. Payne, Single pulse Bragg gratings written during fibre drawing, Electron. Lett. 29(17), 577-1578 (1993)

[7] M. Rothhardt, C. Chojetzki, H. R. Mueller, High mechanical strength single-pulse draw tower gratings, Proc. SPIE Vol. 5579, Part A, 127-135 (2004) 
[8] E.J. Voet, G. Luyckx, I. De Baere, J. Degrieck, J. Vlekken and E. Jacobs, H.Bartelt, High Strain monitoring during Fatigue Loading of Thermoplastic Composites using imbedded draw Tower Fibre Bragg Grating Sensors, Paper Nr. C-4: L16, CIMTEC 2008

[9] L.B. Fu et al., Femtosecond laser writing Bragg gratings in pure silica photonic crystal fibres, Elect. Lett. 41, 638-640 (2005)

[10] T. Geernaert, T. Nasilowski, K. Chah, M. Szpulak, J. Olszewski, G. Statkiewicz, J. Wojcik, K. Poturaj, W. Urbanczyk, M. Becker, M. Rothhardt, H. Bartelt, F. Berghmans, H. Thienpont, Fiber Bragg Gratings in Germanium-doped Highly-Birefringent Microstructured Optical Fibers, IEEE Photonics Technology Letters 20 (8), 554-556 (2008). 